\title{
Two-Phase Multivariate Stratified Sampling with Travel Cost: A Fuzzy Programming Approach
}

\author{
Sana Iftekhar ${ }^{1}$, M.J. Ahsan², Qazi Mazhar Ali ${ }^{3}$,
}

\begin{abstract}
In sample surveys, stratified sampling is useful if the strata weights are known for each stratum. If they are not known double sampling may be used by selecting a large preliminary sample to estimate the strata weights. Then a stratified sample may be selected independently or from the initial sample. If the problem of non-response is also present then the strata are to be virtually divided into two disjoint and exhaustive groups of respondents and non-respondents. A subsamples from non-respondents is then selected and second more extensive attempt is to be group is to be made for obtaining the required information. The problem of obtaining a compromise allocation for first and second phase of sampling is the formulated as a multi-objective nonlinear programming problem that minimizes the sum of variances of the stratified sample mean subject to the non-linear cost constraint. The formulated problem is solved using fuzzy programming and fuzzy goal programming based on piecewise linear approximation. A numerical example is presented to illustrate the computational details.
\end{abstract}

Keywords: Non-response, Multivariate Stratified Sampling, Multiobjective Integer non-linear programming problem, Fuzzy Programming, Travel cost

\section{Introduction}

Our society needs a prompt and accurate flow of information on preferences, need and behavior. It returns a good response on the part of government, business and social institutions. The need of statistical information is rapidly growing in our society. Huge amount of data are being collected and used by the states to plan their economic and social activities, e.g. the effect of sales and production in the business and industrial sectors and the development of research and social projects in scientific institution. A sample survey technique may be used where a part (sample) of the population is evaluated, and inference are drawn about the population as a whole on the basis of this sample.

The 'optimum allocation' in stratified random sampling is well known for a univariate population ( See Cochran (1977) and Sukhatme et al. (1984)) more than one characteristics are defined on each and every unit of the population, it is not feasible to use the individual optimum allocations to the strata unless there is a strong positive correlation between the characteristics under study. Thus, usually, one has to use an allocation that is optimum in 'some sense' for all the characteristics. Such an allocation is known as a compromise allocation in sampling literature. Various compromise criteria to work out a compromise allocation are available. Geary (1949), Dalenius (1957), Ghosh (1958), Yates (1960), Aoyama (1963), Folks and Antle(1965), Chatterjee (1967, 1968), Kokan and Khan (1967), Ahsan (1975-1967, 1978), Ahsan and Khan(1977), Jahan, Khan and Ahsan (1994,2001), Khan, Khan and Ahsan (2003, 2008), Singh (2003), Díaz-García and Cortez (2006, 2008), Kozak (2006a, 2006b), and many others either suggested new compromise criterion or explored further the existing criteria under various situations.

In surveys of human population the problem of non-response is very common. Hansen and Hurwitz (1946) first considered the problem of non-response in sample surveys.

The method of post stratification is useful only if the relative proportion of each stratum in the population $W_{h}\left(=N_{h} / N\right)$ is known for each stratum $h$. If these proportions are not known, double sampling techniques may be used, with an initial (large) sample used to estimate the unknown population parameter $W_{h}$.

Most of the statistician has worked on double sampling problem for stratification where non response is present. Okafor (1994) studied the above problem using a double sampling strategy (DSS) for a univariate stratified population. Najmussehar and Bari (2002) formulated the same problem as a mathematical programming problem and used dynamic programming technique to obtain a solution. Varshney et al (2011) determined a compromise allocation in multivariate stratified random sampling, when strata weights are unknown and non-response is also present. The problem of obtaining a compromise allocation has been formulated as a Multiobjective Integer Nonlinear Programming Problem. The solution is obtained by goal programming technique. Haseen et al. (2012) obtained the solution of this problem by using fuzzy programming techniques.

Generally the real-world decision problems are multiobjective in nature and they conflict with each other regarding optimization of objectives. To resolve the conflict, the goal programming (GP) approach has 
been introduced by Charnes and Cooper (1961). The main problem in using GP is that a precise aspiration level needs to be assigned for each of the objectives. But, in a real-life decision situation, it is difficult to set precise target values to objectives due to imprecise nature of human judgments. To overcome such a situation, fuzzy programming (FP) approach has been introduced by Bellman and Zadeh (1970). Zimmermann first proposed the fuzzy linear programming (1978). In FP, membership functions are defined on the basis of assigned aspiration levels and tolerance ranges defined for the fuzzy goals. But, it is difficult to define tolerance ranges in a highly sensitive decision situation. To overcome such difficulties, goal programming approach in fuzzy environment has been first introduced by Narashimann (1980). Thereafter, Fuzzy Goal Programming (FGP) has been studied by many researchers and has been applied in many real life problems. (See Pal B.B. et al (2003), Biswas et al (2005), Parra et al (2001) etc.).

There are many real-world decision problems in different structural optimization areas. It is found that objectives of most of the industrial problems are nonlinear in nature. To solve such problems, different classical approaches were developed and widely circulated in the literature. One of most widely used approaches is the approximation of a nonlinear function by piecewise linear approximation method.

Separable programming is important because it allows a convex nonlinear program to be approximated with arbitrary accuracy with a linear programming model. Separable programming was first introduced by Miller (1963). Thereafter, it has been developed by Cox (1971), Lin and Chen (2002), Chang (2000), and other researchers. But, separable programming approach in the area of FGP is not widely circulated in literature. Generally, separable programming problems are transformed into linear form by using piecewise linear approximation method.

In sample surveys problem when cost of travelling between the selected units of a stratum is also significant, a cost function quadratic in $\sqrt{n}_{h}$ may be used (See Cochran (1977)). Non linear cost constraint has been studied by many researchers. Shazia Ghufran et al. (2012) worked out the compromise allocation in a multivariate stratified survey using the compromise criterion to minimize the sum of squared coefficients of variation of the estimators subject to the non linear cost. Saman et al. (2013) obtained the compromise solution in presence of non response using travel cost.

In the present manuscript the authors have discussed the problem of Two-Phase sampling (or double sampling) design in presence of non response. The compromise solution of this problem has been obtained by minimizing the variance of double sampling for stratification in presence of non response for fixed travelling cost. The solution obtained using fuzzy programming and fuzzy goal programming technique based on piecewise linear approximation. A numerical example is also given.

\section{Non-response in double sampling for stratification}

A population of size $N$, divided into $L$ strata which are homogenous within themselves. The strata sizes are $N_{1}, N_{2}, \ldots, N_{L}$, where $\sum_{h=1}^{L} N_{h}=N$. The strata weights $W_{h}\left(=N_{h} / N\right)$ are used in estimating unbiasedly the mean or the total of the characteristics under study.If these strata weights are not known the technique of double sampling may be used to estimate them that is, a preliminary simple random sample of size $n^{\prime}$ is selected without replacement, to estimate the strata weights, treating the population as unstratified. Each unit of the sample falling in which stratum is recorded. An unbiased estimate $w_{h}$ is then given by $w_{h}=n_{h}^{\prime} / n^{\prime}$.

Subsample of sizes $n_{h}=v_{h} n_{h}^{\prime} ; h=1,2, \ldots, L ; 0<v_{h} \leq 1$ is then drawn out of $n_{h}^{\prime}$ units using srswor from each stratum for fixed $v_{h}$, to collect information on the characteristics under study, such that $\sum_{h=1}^{L} n_{h}=n$. Let $W_{h}\left(=N_{h} / n\right)$ be proportion of units falling in the $h t h$ strata and $w_{h}\left(=n_{h}^{\prime} / n^{\prime}\right)$ be proportion of first sampling units falling in the $h t h$ stratum. The double sampling for stratification estimator of population mean $\bar{Y}_{j}$ of the $j t h$ characteristic measured on each selected unit is given by

$$
\bar{y}_{h d s}=\sum_{h=1}^{L} w_{h} \bar{y}_{j h}
$$

$\bar{y}_{j h}=\frac{1}{n_{h}} \sum_{i=1}^{n_{h}} y_{j h i}$ is a sample mean of the $j$ th characteristic, where $j=1,2, \ldots, p$ based on $n_{h}$ units for stratum $h$ and 'ds' stand for double sampling.

The sampling variance of $\bar{y}_{j d s}$ is then given by

$$
V\left(\bar{y}_{j d s}\right)=\left(\frac{1}{n^{\prime}}-\frac{1}{N}\right) S_{j}^{2}+\frac{1}{n^{\prime}} \sum_{h=1}^{L} w_{h}\left(\frac{1}{v_{h}}-1\right) S_{j h}^{2}
$$


where $S_{j}^{2}$ and $S_{j h}^{2} \quad$ denote the population and stratum variances of $j t h \quad$ characteristic based on $N$ and $N_{h}$ units.

In the presence of non-response, let $n_{h 1}$ units respond at the first attempt and $n_{h 2}$ units are the number of non-respondents out of $n_{h}$ units. Using Hansen and Hurwitz (1946) technique, subsamples are selected from non-respondents group of sizes $m_{h 2}=k_{h}^{*} n_{h 2} ; 0<k_{h}^{*}<1$ out of $n_{h 2}$ units is drawn and interviewed with extra effort. Where $k_{h}^{*}$ is a known constant $h=1,2, \ldots, L$.

Let $\bar{y}_{j h 1}$ and $\bar{y}_{j m_{h 2}}$ denote the sample mean of $j t h$ characteristic of the $n_{h 1}$ respondents at the first attempt and the $m_{h 2}$ subsampled units at the second attempt.

For $j$ th characteristic, an unbiased estimator $\bar{y}_{j d s}^{*}$ for $\bar{Y}_{j}$ based on sample means from the respondents and the non-respondents group obtained in second attempt is given by

where, $\bar{y}_{j h}^{*}=\frac{n_{h 1} \bar{y}_{j h 1}+n_{h 2} \bar{y}_{j m_{h 2}}}{n_{h}}$

$$
\bar{y}_{j d s}^{*}=\sum_{h=1}^{L} w_{h} \bar{y}_{j h}^{*}
$$

The variance of $\bar{y}_{j d s}^{*}$ is given by

$$
\begin{aligned}
V\left(\bar{y}_{j d s}^{*}\right) & =V\left(\bar{y}_{j d s}\right)+\frac{1}{n^{\prime}} \sum_{h=1}^{L} w_{h 2}\left(\frac{1-k_{h}^{*}}{k_{h}^{*} v_{h}}\right) S_{j h 2}^{2} \\
& =\left(\frac{1}{n^{\prime}}-\frac{1}{N}\right) S_{j}^{2}+\frac{1}{n^{\prime}} \sum_{h=1}^{L} w_{h}\left(\frac{1}{v_{h}}-1\right) S_{j h}^{2}+\frac{1}{n^{\prime}} \sum_{h=1}^{L} w_{h 2}\left(\frac{1-k_{h}^{*}}{k_{h}^{*} v_{h}}\right) S_{j h 2}^{2} \\
& =Z_{j} ; j=1,2, \ldots, p
\end{aligned}
$$

In the above expression $w_{h 2}=n_{h 2} / n_{h}$ is the proportion of non-respondents and $S_{j h 2}^{2}$ is the population variance of $j$ th characteristic, $j=1,2, \ldots, p$ of the non-respondents in the $h t h$ stratum.

A fully generalized cost function draws upon differential calculus and decision theory to minimize variance subject to a given cost (see Cochran, 1963), but a simpler algebraic relationship permits an examination of the effect of sample survey design on survey cost. The cost of carrying out multivariate stratified double sampling in presence of non response are characterized by four major categories as defined in the following.

$$
C=c_{0} n^{\prime}+\sum_{h=1}^{L} c_{h 1} n_{h}+\sum_{h=1}^{L} c_{h 11} n_{h 1}+\sum_{h=1}^{L} c_{h 12} m_{h 2}
$$

where $c_{0}$ is the cost of measuring each unit in the preliminary sample.

$c_{h 1}$ is the cost of measuring each unit in the first attempt (phase-1).

$c_{h 11}$ is the cost of processing the result of all the $p$ characteristic on the $n_{h 1}$ selected units from respondents group in the $h t h$ stratum at phase-I.

$c_{h 12}$ is the cost for measuring and processing the results of all the $p$ characteristic on the $m_{h 2}$ units selected from the non-respondents group in the $h t h$ stratum at the second attempt(phase-II).

$c_{j h 11}$ and $c_{j h 12}$ are the per unit cost of measuring the characteristic at phase-I and phase-II respectively.

Since $n_{h 1}$ is not known until the first attempt has been made, the quantity $w_{h 1} n_{h}$ may be used as its estimated value. The total expected cost of $\hat{C}$ the survey is thus given by

$$
\hat{C}=c_{0} n^{\prime}+\sum_{h=1}^{L}\left(c_{h 1}+w_{h 1} c_{h 11}\right) n_{h}+\sum_{h=1}^{L} c_{h 12} m_{h 2}
$$

In linear cost function the cost associated with the non travel activity of the survey include drawing samples, preparing survey materials, locating identifying and interviewing respondents and coding data. Sometimes, it is not fulfill our requirement. If the cost of travelling between selected units of a stratum is substantial it should also be considered while estimating the total cost of the survey. Then the total cost includes both the travel and the measurement costs. Let the per unit travel cost be $t_{h 1}$ and $t_{h 12}$ for first and second attempt respectively. An adequate approximation to the total expected cost $\hat{C}$ of the double sampling stratified survey in presence of non response will then be

$$
\hat{C}=c_{0} n^{\prime}+\sum_{h=1}^{L}\left(c_{h 1}+w_{h 1} c_{h 11}\right) n_{h}+\sum_{h=1}^{L} c_{h 12} m_{h 2}+\sum_{h=1}^{L} t_{h 1} \sqrt{n_{h}}+\sum_{h=1}^{L} t_{h 12} \sqrt{m_{h 2}}
$$

\section{Formulation of the above problem}

In phase-I, the problem is to find the optimum sizes of the second samples $n_{h} ; h=1,2, \ldots, L$ which may be obtained by minimizing $Z_{j} ; j=1,2, \ldots, p$ given by (4) for the cost given in (7). In phase-II the optimum subsample of size $m_{h 2} ; h=1,2, \ldots, L$ from non response are obtained for a fixed cost of the survey. The multi-objective formulation of the problem at phase-I may be given as 


$$
\left.\begin{array}{ccc}
\text { Minimize } & {\left[Z_{1}, Z_{2}, \ldots, Z_{p}\right]} & \text { simultaneously } \\
\text { Subject to } & \sum_{h=1}^{L}\left(c_{h 1}+w_{h 1} c_{h 11}\right) n_{h}+\sum_{h=1}^{L} c_{h 12} m_{h 2}+\sum_{h=1}^{L} t_{h 1} \sqrt{n_{h}}+\sum_{h=1}^{L} t_{h 12} \sqrt{m_{h 2}} \leq \hat{C}-c_{0} n^{\prime} \\
& 2 \leq n_{h} \leq n_{h}^{\prime} \\
& n_{h} \text { integers } ; h=1,2, \ldots, L
\end{array}\right\}
$$

where $Z_{j} ; j=1,2, \ldots, p$ are as defined in (4).

After ignoring the terms which are independent of $n_{h}$ in (4) and rearranging the cost constraints for first phase, the MINLPP (8) becomes

$$
\begin{aligned}
& \text { Minimize } \quad V_{j}=\sum_{h=1}^{L} \frac{a_{j h}}{n_{h}} \\
& \text { Subject to } \quad \sum_{h=1}^{L}\left(c_{h 1}+w_{h 1} c_{h 11}\right) n_{h}+\sum_{h=1}^{L} t_{h 1} \sqrt{n_{h}} \leq \hat{C}_{0} \\
& 2 \leq n_{h} \leq n_{h}^{\prime} \\
& \text { and } \quad n_{h} \text { integers } ; h=1,2, \ldots, L \\
& \text { where } \quad a_{j h}=\frac{1}{n^{\prime}}\left(\sum_{h=1}^{L} w_{h} n_{h}^{\prime} S_{j h}^{2}+w_{h 2}\left(\frac{\left(1-k_{h}^{*}\right)}{k_{h}^{*}}\right) n_{h}^{\prime} S_{j h 2}^{2}\right) \\
& \text { and } \sum_{h=1}^{L}\left(c_{h 1}+w_{h 1} c_{h 11}\right) n_{h} \leq \hat{C}_{0} \\
& \text { where } \hat{C}_{0}=\hat{C}-c_{0} n^{\prime}-\sum_{h=1}^{L} c_{h 12} m_{h 2}+\sum_{h=1}^{L} t_{h 12} \sqrt{m_{h 2}}
\end{aligned}
$$

Ignoring the terms independent of $m_{h 2}$ in the R.H.S of (4), at phase-II, the problem is to work out the optimum values of $m_{h 2}$ which minimize $V_{j}^{\prime}, j=1,2, \ldots, p$ for a given cost in (7).

Substituting $k_{h}^{*}=m_{h 2} / n_{h 2}$ and $v_{h}=\frac{n_{h}}{n_{h}^{\prime}}$, and rearranging the cost constraints for the phase-II, problem (8) may be stated as

$$
\begin{aligned}
& \text { Minimize } V_{j}^{\prime}=\sum_{h=1}^{L} \frac{b_{j h}}{m_{h 2}} \\
& \text { Subject to } \quad \sum_{h=1}^{L} c_{h 12} m_{h 2}+\sum_{h=1}^{L} t_{h 12} \sqrt{m_{h 2}} \leq \hat{C}_{0}^{\prime} \\
& 2 \leq m_{h 2} \leq n_{h 2} \\
& \begin{array}{c}
m_{h 2} \text { integers } ; h=1,2, \ldots, L ; j=1,2, \ldots, p \\
\text { where } b_{j h}=\frac{1}{n^{\prime}} \sum_{h=1}^{L} \frac{w_{h 2} n_{h 2} n_{h}^{\prime} S_{j h 2}^{2}}{n_{h}}
\end{array} \\
& \text { and } \hat{C}_{0}^{\prime}=\hat{C}-c_{0} n^{\prime}-\sum_{h=1}^{L}\left(c_{h 1}+w_{h 1} c_{h 11}\right) n_{h}+\sum_{h=1}^{L} t_{h 1} \sqrt{n_{h}} \text {. }
\end{aligned}
$$

\section{The Solution}

In the following two approaches to solve to solve the formulated problems in phase-I and phase-II are discussed. The first approach is through Fuzzy Programming Technique while the second approach is through Fuzzy Goal Programming Technique using piecewise linear approximation.

\subsection{Fuzzy Programming}

Let $V_{j}^{*}$ is the optimal value of $V_{j}$ obtained by solving the MINLPP (9)

Further let

$$
\tilde{V}_{j}=\tilde{V}_{j}\left(n_{1}, n_{2}, \ldots, n_{h}, \ldots, n_{L}\right)
$$

denote the value of the variances under the compromise allocation, where $n_{h} ; h=1,2, \ldots, L$ are to be worked out. 
Obviously $\tilde{V}_{j} \geq V_{j}^{*}$ and $\tilde{V}_{j}-V_{j}^{*} \geq 0 ; j=1,2, \ldots, p$ will give the increase in the variance due to not using the individual optimum allocation for $j^{\text {th }}$ characteristic.

To obtain a fuzzy solution, we first compute the maximum value $U_{j}$ and the minimum value $L_{j}$, for each $j=1,2, \ldots, p$.

Now,

$$
L_{j}=\min _{j} V\left(n_{h, j}^{*}\right) \quad U_{j}=\max _{j} V\left(n_{h, j}^{*}\right)
$$

where $n_{h, j}^{*}$ denote the optimum allocation for the $j^{\text {th }}$ characteristic in four strata.

The differences of the maximum and minimum values of $V_{j}$ are denoted by $d_{j}=U_{j}-L_{j}, j=1,2, \ldots, p$.

The fuzzy programming formulation of the MINLPP in (9) is given by the following INLPP:

$$
\begin{aligned}
& \text { Minimize } \delta \\
& \text { Subject to } \\
& \sum_{h=1}^{L} \frac{a_{j h}}{n_{h}}-\delta d_{k} \leq V_{j}^{*} \\
& \sum_{h=1}^{L}\left(c_{h 1}+w_{h 1} c_{h 11}\right) n_{h}+\sum_{h=1}^{L} t_{h 1} \sqrt{n_{h}} \leq \hat{C}_{0} \\
& 2 \leq n_{h} \leq n_{h}^{\prime} n_{h} \geq 0, \delta \geq 0, \\
& \text { and } \quad n_{h} \text { are integers; } \quad j=1,2, \ldots p ; h=1,2, \ldots, L
\end{aligned}
$$

where $\delta \geq 0$ is the decision variable representing the worst deviation level.

The fuzzy programming (FPP) may be solved using the optimization software LINGO-13.

Similarly, for Phase II the MINLPP (10) FPP becomes

$$
\begin{array}{ll}
\text { Minimize } & \delta^{\prime} \\
\text { Subject to } & \sum_{h=1}^{L} \frac{b_{j h}}{m_{h 2}}-\delta^{\prime} d_{k} \leq V_{j}^{\prime *} \\
& \sum_{h=1}^{L} c_{h 12} m_{h 2}+\sum_{h=1}^{L} t_{h 12} \sqrt{m_{h 2}} \leq \hat{C}_{0}^{\prime} \\
& 2 \leq m_{h 2} \leq n_{h 2}, \delta^{\prime} \geq 0, \\
\text { and } \quad m_{h 2} \text { are integers } ; \quad j=1,2, \ldots p ; h=1,2, \ldots, L
\end{array}
$$

\subsection{Fuzzy Goal Programming using piecewise linear approximation}

First, we formulate the fuzzy programming model of NLLP in (8) by transforming the objective function $V_{j}$ into fuzzy goals by means of assigning an imprecise aspiration level for each objective. Let $V_{j}^{*}$ be the optimal solution of the objective function. Then the fuzzy goals appear in the form $V_{1} \simeq V_{1}^{*}$

\section{$V_{j} \stackrel{\check{\Sigma}}{V_{j}^{*}}$}

Using the individual best solution, we find the upper and lower tolerance limit $U_{j}$ and $L_{j}$ for each objective function already described in section (4.1).

The fuzzy goals are characterized by their membership functions. The membership function of objective function for each characteristic is given below

$$
\mu_{j}(\bar{n})=\left\{\begin{array}{cl}
1 & \text { if } V_{j}(\bar{n}) \leq L \\
\frac{U_{j}-V_{j}(\bar{n})}{U_{j}-L_{j}} & \text { if } L_{j} \leq V_{j}(\bar{n}) \leq U_{j}, \\
0 & \text { if } V_{j}(\bar{n}) \geq U_{j},
\end{array}\right\} j=1,2, \ldots, p
$$

In the fuzzy goal programming (FGP) formulation, the defined membership function in equation (15) for each characteristics are transformed into membership goals by introducing under and over deviational variables and assigning the highest membership value means unity (one) as the aspiration level to each of them. Under the above circumstances, the membership goals are

$$
\frac{U_{j}-V_{j}(\bar{n})}{U_{j}-L_{j}}+d_{j}=1 ; j=1,2, \ldots, p
$$

Where $d_{j} \geq 0$ represent the under and over deviational variables.

We transform the non linear membership function $\mu_{j}(\bar{n})$ into equivalent linear membership function by using piecewise linear approximation: 
The goal in (16) can be written as

$$
\frac{U_{j}-\sum_{h=1}^{L} f_{j h}\left(n_{h}\right)}{U_{j}-L_{j}}+d_{j}=1 ; j=1,2, \ldots, p \quad h=1,2, \ldots, L
$$

For linearizing the non linear function $f_{j h}\left(n_{h}\right)$ in (17), the grid points for the variable $n_{h}(h=1,2, \ldots L)$ are chosen as $a_{h r}\left(r=0,1, \ldots, r_{h}\right)$ Further, we introduced a new variable $y_{h r}\left(r=0,1, \ldots, r_{h}\right)$, so that $n_{h}$ can be expressed as

$$
n_{h}=\sum_{r=0}^{r_{h}} a_{h r} y_{h r}
$$

Where $\sum_{r=0}^{r_{h}} y_{h r}=1\left(y_{h r} \geq 0\right)$ with grid points $a_{h 0}, a_{h 1}, \ldots, a_{h r_{h}}$.

So, the piecewise linear form of nonlinear function $f_{j h}\left(n_{h}\right)$ nominated as $F_{j h}$ can be written as

$$
F_{j h}=\sum_{r=0}^{r_{h}} y_{h r} f_{j h}\left(a_{h r}\right)
$$

Then, the linear FGP model by using expression of (18) and (19) in MINLPP (9) for Phase I can Minimize $Z=\sum_{j=1}^{p} d_{j}$

so as to satisfy

$$
\begin{gathered}
\frac{1}{U_{j}-L_{j}}\left[U_{j}-\sum_{h=1}^{L} F_{j h}\right]+d_{j}=1 \\
\text { where } F_{j h}=\sum_{r=0}^{r_{h}} y_{h r} f_{j h}\left(a_{h r}\right)
\end{gathered}
$$

Subject to $\quad \sum_{h=1}^{L}\left(c_{h 1}+w_{h 1} c_{h 11}\right) \sum_{r=0}^{r_{h}} a_{h r} y_{h r}+\sum_{h=1}^{L} t_{h 1} \sqrt{\sum_{r=0}^{r_{h}} a_{h r} y_{h r}} \leq \hat{C}_{0}$

$$
\begin{gathered}
\sum_{r=0}^{r_{k}} \gamma_{k r}=1\left(\gamma_{k r} \geq 0\right) \\
h=1,2, \ldots L \\
r=0,1, \ldots, r_{k} \\
j=1,2, \ldots, p
\end{gathered}
$$

Simlarly, for Phase- II FGPP for MINLPP (10) can be written as

$$
\operatorname{Minimize} Z=\sum_{j=1}^{p} d_{j}^{\prime}
$$

so as to satisfy

$$
\frac{1}{U_{j}-L_{j}}\left[U_{j}-\sum_{h=1}^{L} F_{j h}^{\prime}\right]+d_{j}^{\prime}=1
$$

where

$$
F_{j h}^{\prime}=\sum_{r=0}^{r_{h}} y_{h r} f_{j h}\left(a_{h r}\right)_{j h}^{\prime}
$$

Subject to $\sum_{h=1}^{L} c_{h 12} \sum_{r=0}^{r_{h}} a_{h r} y_{h r}+\sum_{h=1}^{L} t_{h 12} \sqrt{\sum_{r=0}^{r_{h}} a_{h r} y_{h r}} \leq \hat{C}_{0}^{\prime}$

$$
\begin{gathered}
\sum_{r=0}^{r_{k}} \gamma_{k r}=1\left(\gamma_{k r} \geq 0\right) \\
h=1,2, \ldots L \\
r=0,1, \ldots, r_{k} \\
j=1,2, \ldots, p
\end{gathered}
$$

In above expression $m_{h 2}=\sum_{r=0}^{r_{h}} a_{h r} y_{h r}$

\section{Numerical Example}

An application of the above technique is given in the following using a numerical data of Haseen et al (2011). The travelling costs $t_{h 1}$ and $t_{h 12}$ are assumed by authors.

A population of size $N=3850$ is divided into four strata. Two characteristics are defined on each unit of the population. It is assumed that the estimation of population means of the two characteristics is of interest. 
Table 1 shows the required information. Each stratum is further subdivided into respondents and nonrespondents groups as given in Table2. It is assumed that $v_{h}$ and $k_{h}^{*}$ are known and the preliminary sample size $n^{\prime}=1000$.

Table 1 Data for four strata and two characteristics

\begin{tabular}{|l|l|l|l|l|l|l|l|l|l|l|}
\hline$h$ & $w_{h}$ & $S_{1 h}^{2}$ & $S_{2 h}^{2}$ & $v_{h}$ & $k_{h}^{*}$ & $c_{h 1}$ & $c_{h 11}$ & $c_{h 12}$ & $t_{h 1}$ & $t_{h 12}$ \\
\hline \hline 1 & 0.32 & 784 & 1444 & 0.4 & 0.5 & 1 & 2 & 3 & 0.5 & 2 \\
\hline 2 & 0.21 & 576 & 676 & 0.5 & 0.6 & 1 & 3 & 4 & 0.5 & 2.5 \\
\hline 3 & 0.27 & 1024 & 1936 & 0.6 & 0.7 & 1 & 4 & 5 & 0.5 & 3 \\
\hline 4 & 0.20 & 2916 & 6084 & 0.65 & 0.75 & 1 & 5 & 6 & 0.5 & 4.5 \\
\hline
\end{tabular}

Table 2 Subdivided data as respondents and non-respondents groups for four strata with two characteristics

\begin{tabular}{|c|c|c|c|c|}
\hline$h$ & Group & $S_{1 h}^{2}$ & $S_{2 h}^{2}$ & $\begin{array}{c}w_{h k} \\
k=1,2\end{array}$ \\
\hline \multirow{2}{*}{1} & Respondents & 361.06 & 767.82 & $w_{11}=0.70$ \\
\hline & Non-respondents & 310.55 & 454.76 & $w_{12}=0.30$ \\
\hline \multirow{2}{*}{2} & Respondents & 373.79 & 449.92 & $w_{21}=0.80$ \\
\hline & Non-respondents & 326.29 & 353.81 & $w_{22}=0.20$ \\
\hline \multirow{2}{*}{3} & Respondents & 930.15 & 1272.88 & $w_{31}=0.75$ \\
\hline & Non-respondents & 560.28 & 1165.97 & $w_{32}=0.25$ \\
\hline \multirow{2}{*}{4} & Respondents & 2355.98 & 2690.53 & $w_{41}=0.72$ \\
\hline & Non-respondents & 1013.08 & 2403.55 & $w_{42}=0.28$ \\
\hline
\end{tabular}

In the last column of the Table $2, k=1$ is for respondents group and $k=2$ is for non-respondents group. Further the total amount available for the survey be $C=3000$ units. Out of these 3000 units 750 units are earmarked for the preliminary sample of size $n^{\prime}, 1,900$ units are earmarked for phase-I and 350 units are earmarked for phase-II.

Using estimated values of strata weights and the size of selected preliminary sample, the values of $n_{h}^{\prime}=$ $w_{h} n^{\prime} ; h=1,2, \ldots, L$ are obtained as

$$
n_{1}^{\prime}=320, n_{2}^{\prime}=210, n_{3}^{\prime}=270, n_{4}^{\prime}=200 \text { with } \sum_{h=1}^{4} n_{h}^{\prime}=1,000 .
$$

\subsection{Solution using Fuzzy programming}

We obtain the individual best solution for each of the objective in Phase I. After putting the values from Table 1 and Table 2, the MINLPP (9) becomes

For $j=1$

$$
\begin{array}{ll}
\text { Minimize } & V_{1}=\frac{110.09440}{n_{1}}+\frac{45.95787}{n_{2}}+\frac{162.89370}{n_{3}}+\frac{286.83744}{n_{4}} \\
\text { Subject to } & 2.4 n_{1}+3.4 n_{2}+4 n_{3}+4.6 n_{4}+0.5 \sqrt{n_{1}}+0.5 \sqrt{n_{2}}+0.5 \sqrt{n_{3}}+0.5 \sqrt{n_{4}} \leq 1900 \\
2 & \leq n_{1} \leq 320 \\
2 & \leq n_{2} \leq 210 \\
2 & \leq n_{3} \leq 270 \\
2 & \leq n_{4} \leq 200 \\
\text { and } & n_{h} \text { integers } ; h=1,2, \ldots, 4 .
\end{array}
$$

The optimum solution of the above problem using software LINGO-13 is

Similarly, for $j=2$

$$
n_{11}^{*}=140, n_{21}^{*}=75, n_{31}^{*}=133, n_{41}^{*}=164 \text { with } V_{1}^{*}=4.372934
$$

$$
\begin{aligned}
& \text { Minimize } V_{1}=\frac{191.52260}{n_{1}}+\frac{52.10163}{n_{2}}+\frac{324.7747}{n_{3}}+\frac{647.15640}{n_{4}} \\
& \text { Subject to } \begin{aligned}
2.4 n_{1}+3.4 n_{2}+4 n_{3}+4.6 n_{4}+0.5 \sqrt{n_{1}}+0.5 \sqrt{n_{2}}+0.5 \sqrt{n_{3}}+0.5 \sqrt{n_{4}} \leq 1900 \\
2 \leq n_{1} \leq 320 \\
2 \leq n_{2} \leq 210 \\
2 \leq n_{3} \leq 270 \\
2 \leq n_{4} \leq 200 \\
\text { and } \\
n_{h} \text { integers } ; h=1,2, \ldots, 4 .
\end{aligned}
\end{aligned}
$$

The optimum solution using software LINGO-13 is

$$
n_{11}^{*}=136, n_{21}^{*}=58, n_{31}^{*}=135, n_{41}^{*}=177 \quad \text { with } V_{2}^{*}=8.368548
$$

Further the value of $V_{1}$ at the point $n_{h 2}^{*}$ is 4.429065 and the value of $V_{2}$ at the point $n_{h 1}^{*}$ is 8.450698 . Thus,

$$
L_{1}=4.372934, \quad U_{1}=4.429065
$$




$$
\begin{aligned}
L_{2} & =8.368548, & U_{2} & =8.450698 \\
d_{1} & =0.056131, & d_{2} & =0.08215
\end{aligned}
$$

After computing the optimum allocation and optimal variances for the two characteristics the compromising optimal solution for the above problem can be obtained by solving the given FPP (13)

$$
\begin{aligned}
& \text { Minimize } \delta \\
& \text { Subject to } \frac{110.09440}{n_{1}}+\frac{45.95787}{n_{2}}+\frac{162.89370}{n_{3}}+\frac{286.83744}{n_{4}}-\delta(0.056131) \leq 4.372934 \\
& \frac{191.52260}{n_{1}}+\frac{52.10163}{n_{2}}+\frac{324.7747}{n_{3}}+\frac{647.15640}{n_{4}}-\delta(0.08215) \leq 8.368548 \\
& 2.4 n_{1}+3.4 n_{2}+4 n_{3}+4.6 n_{4}+0.5 \sqrt{n_{1}}+0.5 \sqrt{n_{2}}+0.5 \sqrt{n_{3}}+0.5 \sqrt{n_{4}} \leq 1900 \\
& 2 \leq n_{1} \leq 320 \\
& 2 \leq n_{2} \leq 210 \\
& 2 \leq n_{3} \leq 270 \\
& 2 \leq n_{4} \leq 200 \\
& n_{h} \text { integers } ; h=1,2, \ldots, 4 \text {. }
\end{aligned}
$$

Now the fuzzy compromise solution using LINGO-13 is

Similarly for Phase II the MINLPP (10)

$$
\begin{gathered}
n_{1}^{*}=136, n_{2}^{*}=67, n_{3}^{*}=133, n_{4}^{*}=172 \text { with } \delta=0.2662736 \\
V_{1}=4.387880, \quad V_{2}=8.390343
\end{gathered}
$$

For $j=1$

$$
\begin{aligned}
& \text { Minimize } V_{1}^{\prime}=\frac{8.94384}{m_{12}}+\frac{2.740836}{m_{22}}+\frac{9.454725}{m_{32}}+\frac{15.88509}{m_{42}} \\
& \text { Subject to } \quad \begin{aligned}
3 m_{12}+4 m_{22}+5 m_{32} & +6 m_{42}+2 \sqrt{m_{12}}+2.5 \sqrt{m_{22}}+3 \sqrt{m_{32}}+4.5 \sqrt{m_{42}} \leq 350 \\
2 & \leq m_{12} \leq 41 \\
2 & \leq m_{22} \leq 12 \\
2 & \leq m_{32} \leq 34 \\
2 & \leq m_{42} \leq 50
\end{aligned} \\
& \text { and } \\
& m_{h 2} \text { integers } ; h=1,2, \ldots, 4 .
\end{aligned}
$$

The optimum solution of the above problem using LINGO-13 is

$$
m_{1,12}^{*}=20, m_{1,22}^{*}=9, m_{1,32}^{*}=17, m_{1,42}^{*}=20 \quad \text { with } V_{1}^{\prime *}=2.102144
$$

Similarly, for $j=2$

$$
\begin{aligned}
& \text { Minimize } V_{2}^{\prime}=\frac{191.52260}{m_{12}}+\frac{52.10163}{m_{22}}+\frac{324.7747}{m_{32}}+\frac{647.15640}{m_{42}} \\
& \text { Subject to } \quad 3 m_{12}+4 m_{22}+5 m_{32}+6 m_{42}+2 \sqrt{m_{12}}+2.5 \sqrt{m_{22}}+3 \sqrt{m_{32}}+4.5 \sqrt{m_{42}} \leq 350 \\
& 2 \leq m_{12} \leq 41 \\
& 2 \leq m_{22} \leq 12 \\
& 2 \leq m_{32} \leq 34 \\
& 2 \leq m_{42} \leq 50 \\
& \text { and } \\
& m_{h 2} \text { integers } ; h=1,2, \ldots, 4 .
\end{aligned}
$$

The optimum solution by using software LINGO-13 is

$$
m_{2,12}^{*}=17, m_{2,22}^{*}=7, m_{2,32}^{*}=18, m_{2}^{*} 42=22 \quad \text { with } V_{2}^{*}=4.001161
$$

Further the value of $V_{1}^{\prime}$ at the point $m_{2, h 2}^{*}$ is 2.164968 and the value of $V_{2}^{\prime}$ at the point $m_{2, h 2}^{*}$ is 4.026857 . Thus,

$$
\begin{array}{ll}
L_{1}=2.102144, & U_{1}=2.164968 \\
L_{2}=4.001161, & U_{2}=4.026857 \\
d_{1}=0.062824, & d_{2}=0.025696
\end{array}
$$

After computing the optimum allocation and optimal variances for the two characteristics the compromising optimal solution for the above problem can be obtained by solving the given FPP (14) 
Minimize

Subject to

$$
\begin{gathered}
\frac{8.94384}{m_{12}}+\frac{2.740836}{m_{22}}+\frac{9.454725}{m_{32}}+\frac{15.88509}{m_{42}} \\
\delta^{\prime}(0.0628241) \leq 2.102144 \\
\frac{191.52260}{m_{12}}+\frac{52.10163}{m_{22}}+\frac{324.7747}{m_{32}}+\frac{647.15640}{m_{42}}
\end{gathered}
$$$$
\delta^{\prime}(0.025696) \leq 4.001161
$$$$
3 m_{12}+4 m_{22}+5 m_{32}+6 m_{42}+
$$$$
2 \sqrt{m_{12}}+2.5 \sqrt{m_{22}}+3 \sqrt{m_{32}}+4.5 \sqrt{m_{42}} \leq 350
$$$$
2 \leq m_{12} \leq 41
$$$$
2 \leq m_{22} \leq 12
$$$$
2 \leq m_{32} \leq 34
$$$$
2 \leq m_{42} \leq 50
$$

and $m_{h 2}$ integers $; h=1,2, \ldots, 4$

Now the fuzzy compromise solution by using software LINGO 13 is

$$
\begin{gathered}
m_{12}^{*}=18, m_{22}^{*}=9, m_{32}^{*}=17, m_{42}^{*}=21 \quad \text { with } \delta^{\prime}=0.3395363 \\
V_{1}^{\prime}=2.114010, \quad V_{2}^{\prime}=4.009886
\end{gathered}
$$

5.2 Fuzzy goal programming using piecewise linear approximation

For Phase-I, we calculate the membership function for each characteristic

$$
\begin{aligned}
\mu_{1}(\bar{n}) & =\frac{1}{0.056131}\left[4.429065-V_{1}(\bar{n})\right] \\
\text { and } \quad \mu_{2}(\bar{n}) & =\frac{1}{0.08215}\left[8.450698-V_{2}(\bar{n})\right]
\end{aligned}
$$

Each of the objective function can be expressed as the sum of the separable functions which are shown in the table 3.

Table 3 Separable functions associated with the each objective

\begin{tabular}{|c|c|}
\hline$f_{11}\left(n_{1}\right)$ & $110.09440 / n_{1}$ \\
\hline$f_{12}\left(n_{2}\right)$ & $45.95787 / n_{2}$ \\
\hline$f_{13}\left(n_{3}\right)$ & $162.89370 / n_{3}$ \\
\hline$f_{14}\left(n_{4}\right)$ & $286.83744 / n_{4}$ \\
\hline$f_{21}\left(n_{1}\right)$ & $191.52260 / n_{1}$ \\
\hline$f_{22}\left(n_{2}\right)$ & $52.10163 / n_{2}$ \\
\hline$f_{23}\left(n_{3}\right)$ & $324.7747 / n_{3}$ \\
\hline$f_{24}\left(n_{4}\right)$ & $647.15640 / n_{4}$ \\
\hline
\end{tabular}

After introducing the deviational variables the nonlinear membership goals are

$$
\begin{aligned}
& \frac{1}{0.056131}\left[4.429065-f_{11}\left(n_{1}\right)-f_{12}\left(n_{2}\right)-f_{13}\left(n_{3}\right)-f_{14}\left(n_{4}\right)\right]+d_{1}=1 \\
& \text { and } \frac{1}{0.08215}\left[8.450698-f_{21}\left(n_{1}\right)-f_{22}\left(n_{2}\right)-f_{23}\left(n_{3}\right)-f_{24}\left(n_{4}\right)\right]+d_{2}=1
\end{aligned}
$$

These non linear membership goals are approximated with linear form. Let the grid points for each variables $n_{1}, n_{2}, n_{3}, n_{4}$ are

$$
\begin{gathered}
a_{10}=2, a_{11}=81.5, a_{12}=161, a_{13}=240.5, a_{14}=320 \\
a_{20}=2, a_{21}=54, a_{22}=106, a_{23}=158, a_{24}=210 \\
a_{30}=2, a_{31}=69, a_{32}=136, a_{33}=203, a_{34}=270 \\
a_{40}=2, a_{41}=51.5, a_{42}=101, a_{43}=150.5, a_{44}=200
\end{gathered}
$$

The piecewise linear approximation to the function given in table 3 are

$$
\begin{gathered}
F_{11}=(55.04720) y_{10}+(1.350852) y_{11}+(0.6838161) y_{12}+(0.4577730) y_{13}+(0.3440450) y_{14} \\
F_{12}=(22.97893) y_{20}+(0.8510717) y_{21}+(0.4335648) y_{22}+(0.2908726) y_{23}+(0.2188470) y_{24} \\
F_{13}=(81.44685) y_{30}+(2.360778) y_{31}+(1.197748) y_{32}+(0.8004604) y_{33}+(0.6033100) y_{34} \\
F_{14}=(143.4187) y_{40}+(5.569659) y_{41}+(2.839975) y_{42}+(1.905897) y_{43}+(1.434187) y_{44} \\
F_{21}=(95.76130) y_{10}+(2.349971) y_{11}+(1.189581) y_{12}+(0.7963518) y_{13}+(0.5985081) y_{14} \\
F_{22}=(26.050821) y_{20}+(0.9648450) y_{21}+(0.4915248) y_{22}+(0.3297572) y_{23}+(0.2481030) y_{24} \\
F_{23}=(162.3873) y_{30}+(4.706880) y_{31}+(2.388049) y_{32}+(1.595944) y_{33}+(1.202869) y_{34} \\
F_{24}=(323.5782) y_{40}+(12.56614) y_{41}+(6.407489) y_{42}+(4.300043) y_{43}+(3.235782) y_{44} \\
g_{1}=2 y_{10}+81.5 y_{11}+161 y_{12}+240.5 y_{13}+320 y_{14}
\end{gathered}
$$




$$
\begin{aligned}
& g_{2}=2 y_{20}+54 y_{21}+106 y_{22}+158 y_{23}+210 y_{24} \\
& g_{3}=2 y_{30}+69 y_{31}+136 y_{32}+203 y_{33}+270 y_{34} \\
& g_{4}=2 y_{40}+51.5 y_{41}+101 y_{42}+150.5 y_{43}+200 y_{44}
\end{aligned}
$$

Using these values the proposed linear FGP at Phase-I becomes

$$
\begin{aligned}
& \text { Minimize } V=d_{1}+d_{2} \\
& \text { Subject to } \frac{1}{0.056131}\left[4.429065-F_{11}-F_{12}-F_{13}-F_{14}\right]+d_{1}=1 \\
& \frac{1}{0.08215}\left[8.450698-F_{21}-F_{22}-F_{23}-F_{24}\right]+d_{2}=1 \\
& 2.4 g_{1}+3.4 g_{2}+4 g_{3}+4.6 g_{4}+0.5 \sqrt{g_{1}}+0.5 \sqrt{g_{2}}+0.5 \sqrt{g_{3}}+0.5 \sqrt{g_{4}} \leq 1900 \\
& \gamma_{10}+\gamma_{11}+\gamma_{12}+\gamma_{13}+\gamma_{14}=1 \\
& \gamma_{20}+\gamma_{21}+\gamma_{22}+\gamma_{23}+\gamma_{24}=1 \\
& \gamma_{30}+\gamma_{31}+\gamma_{32}+\gamma_{33}+\gamma_{34}=1 \\
& \gamma_{40}+\gamma_{41}+\gamma_{42}+\gamma_{43}+\gamma_{44}=1 \\
& \gamma_{k r} \geq 0 ; k=1,2,3,4 \text { and } r=0,1,2,3,4
\end{aligned}
$$

Using the software LINGO-13 the problem is solved and the obtained solution is

$$
\begin{gathered}
n_{1}^{*}=161, n_{2}^{*}=54, n_{3}^{*}=136, n_{4}^{*}=163 \text { with } V_{1}=4.492375 \text { and } V_{2}=8.512760 \\
C=1886.033
\end{gathered}
$$

Similarly, Phase-II, we calculate the membership function for each characteristic

$$
\begin{aligned}
\mu_{1}(\bar{m}) & =\frac{1}{0.062824}\left[2.164968-V_{1}^{\prime}(\bar{m})\right] \\
\text { and } \quad \mu_{2}(\bar{m}) & =\frac{1}{0.025696}\left[4.026857-V_{2}^{\prime}(\bar{m})\right]
\end{aligned}
$$

Each of the objective function can be expressed as the sum of the separable functions which are shown in the table 4.

Table 4 Separable functions associated with the each objective

\begin{tabular}{|c|c|}
\hline$f_{11}^{\prime}\left(m_{12}\right)$ & $8.94384 / m_{12}$ \\
\hline$f_{12}^{\prime}\left(m_{22}\right)$ & $2.740836 / m_{22}$ \\
\hline$f_{13}^{\prime}\left(m_{32}\right)$ & $9.454725 / m_{32}$ \\
\hline$f_{14}^{\prime}\left(m_{42}\right)$ & $15.88509 / m_{42}$ \\
\hline$f_{21}^{\prime}\left(m_{12}\right)$ & $13.09709 / m_{12}$ \\
\hline$f_{22}^{\prime}\left(m_{22}\right)$ & $2.972004 / m_{22}$ \\
\hline$f_{23}^{\prime}\left(m_{32}\right)$ & $19.67574 / m_{32}$ \\
\hline$f_{24}^{\prime}\left(m_{42}\right)$ & $37.68766 / m_{42}$ \\
\hline
\end{tabular}

After introducing the deviational variables the nonlinear membership goals are

$$
\begin{aligned}
& \frac{1}{0.062824}\left[2.164968-f_{11}^{\prime}\left(m_{12}\right)-f_{12}^{\prime}\left(m_{22}\right)-f_{13}^{\prime}\left(m_{32}\right)-f_{14}^{\prime}\left(m_{42}\right)\right]+d_{1}^{\prime}=1 \\
& \text { and } \frac{1}{0.025696}\left[4.026857-f_{21}^{\prime}\left(m_{12}\right)-f_{22}^{\prime}\left(m_{22}\right)-f_{23}^{\prime}\left(m_{32}\right)-f_{24}^{\prime}\left(m_{42}\right)\right]+d_{2}^{\prime}=1
\end{aligned}
$$

These non linear membership goals are approximated with linear form. Let the grid points for each subsample $m_{12}, m_{22}, m_{32}, m_{42}$ are

$$
\begin{gathered}
a_{10}=2, a_{11}=11.75, a_{12}=21.5, a_{13}=31.25, a_{14}=41 \\
a_{20}=2, a_{21}=4.5, a_{22}=7, a_{23}=9.5, a_{24}=12 \\
a_{30}=2, a_{31}=10, a_{32}=18, a_{33}=26, a_{34}=34 \\
a_{40}=2, a_{41}=14, a_{42}=26, a_{43}=38, a_{44}=50
\end{gathered}
$$

The piecewise linear approximation to the function given in table 4 are

$$
\begin{gathered}
F_{11}^{\prime}=(4.471920) y_{10}+(0.7611779) y_{11}+(0.4159926) y_{12}+(0.2862029) y_{13}+(0.2181424) y_{14} \\
F_{12}^{\prime}=(1.370418) y_{20}+(0.6090747) y_{21}+(0.3915480) y_{22}+(0.2885091) y_{23}+(0.2284030) y_{24} \\
F_{13}^{\prime}=(4.727362) y_{30}+(0.9454725) y_{31}+(0.5252625) y_{32}+(0.3636433) y_{33}+(0.2626312) y_{34} \\
F_{14}^{\prime}=(7.942545) y_{40}+(1.134649) y_{41}+(0.6109650) y_{42}+(0.4180287) y_{43}+(0.3177018) y_{44} \\
F_{21}^{\prime}=(6.548545) y_{10}+(1.114646) y_{11}+(0.6091670) y_{12}+(0.4191069) y_{13}+(0.3194412) y_{14} \\
F_{22}^{\prime}=(1.486002) y_{20}+(0.6604453) y_{21}+(0.4245720) y_{22}+(0.3128425) y_{23}+(0.2476670) y_{24} \\
F_{23}^{\prime}=(9.837870) y_{30}+(1.967574) y_{31}+(1.093097) y_{32}+(0.7567592) y_{33}+(0.5465483) y_{34} \\
F_{24}^{\prime}=(18.84383) y_{40}+(2.691976) y_{41}+(1.449525) y_{42}+(0.9917805) y_{43}+(0.7537532) y_{44}
\end{gathered}
$$




$$
\begin{gathered}
g_{1}^{\prime}=2 y_{10}+11.75 y_{11}+21.5 y_{12}+31.25 y_{13}+41 y_{14} \\
g_{2}^{\prime}=2 y_{20}+4.5 y_{21}+7 y_{22}+9.5 y_{23}+12 y_{24} \\
g_{3}^{\prime}=2 y_{30}+10 y_{31}+18 y_{32}+26 y_{33}+34 y_{34} \\
g_{4}^{\prime}=2 y_{40}+14 y_{41}+26 y_{42}+38 y_{43}+50 y_{44}
\end{gathered}
$$

Using these values the proposed linear FGP at Phase-II becomes

$$
\begin{array}{cl}
\text { Minimize } & V^{\prime}=d_{1}^{\prime}+d_{2}^{\prime} \\
\text { Subject to } & \frac{1}{0.062824}\left[2.164968-F_{11}^{\prime}-F_{12}^{\prime}-F_{13}^{\prime}-F_{14}^{\prime}\right]+d_{1}^{\prime}=1 \\
\frac{1}{0.025696}\left[4.026857-F_{21}-F_{22}-F_{23}-F_{24}\right]+d_{2}^{\prime}=1 \\
3 g_{1}^{\prime}+4 g_{2}^{\prime}+5 g_{3}^{\prime}+6 g_{4}+2 \sqrt{g_{1}}+2.5 \sqrt{g_{2}}+3 \sqrt{g_{3}}+4.5 \sqrt{g_{4}^{\prime}} \leq 350 \\
\gamma_{10}+\gamma_{11}+\gamma_{12}+\gamma_{13}+\gamma_{14}=1 \\
\gamma_{20}+\gamma_{21}+\gamma_{22}+\gamma_{23}+\gamma_{24}=1 \\
\gamma_{30}+\gamma_{31}+\gamma_{32}+\gamma_{33}+\gamma_{34}=1 \\
\gamma_{40}+\gamma_{41}+\gamma_{42}+\gamma_{43}+\gamma_{44}=1 \\
\gamma_{k r} \geq 0 ; k=1,2,3,4 \text { and } r=0,1,2,3,4,
\end{array}
$$

\begin{tabular}{|c|c|c|c|c|c|c|c|}
\hline \multirow[b]{2}{*}{ Techniques } & \multicolumn{4}{|c|}{ Allocations } & \multicolumn{2}{|l|}{ Variances } & \multirow{2}{*}{$\begin{array}{l}\text { Cost } \\
\text { incurred }\end{array}$} \\
\hline & $n_{1}$ & $n_{2}$ & $n_{3}$ & $n_{4}$ & $V_{1}$ & $V_{2}$ & \\
\hline FP & 136 & 67 & 133 & 172 & 4.387880 & 8.390343 & 1900 \\
\hline FGP & 161 & 54 & 136 & 163 & 4.492375 & 8.512760 & 1886 \\
\hline
\end{tabular}

\begin{tabular}{|c|c|c|c|c|c|c|c|}
\hline \multirow[t]{2}{*}{ Techniques } & \multicolumn{4}{|c|}{ Allocations } & \multicolumn{2}{|l|}{ Variances } & \multirow{2}{*}{$\begin{array}{l}\text { Cost } \\
\text { incurred }\end{array}$} \\
\hline & $m_{12}$ & $m_{22}$ & $m_{32}$ & $m_{42}$ & $V_{1}^{\prime}$ & $V_{2}^{\prime}$ & \\
\hline FP & 18 & 9 & 17 & 21 & 2.114010 & 4.009886 & 350 \\
\hline FGP & 12 & 7 & 18 & 24 & 2.324009 & 4.179412 & 346 \\
\hline
\end{tabular}

Using the software LINGO-13 the problem is solved and the obtained solution is

$$
m_{12}^{*}=12, m_{22}^{*}=7, m_{32}^{*}=18, m_{42}^{*}=24 \quad \text { with } V_{1}=2.324009 \text { and } V_{2}=4.179412
$$

and $C=346.3159$

Table 5 Solution at Phase-I

Table 6 Solution at Phase-II

\section{Conclusion}

Tables 5 and 6 show that none of the two methods is uniformly better than the other. At Phase-I the Fuzzy Goal Programming (FGP) technique gives a slightly better result as compared to the Fuzzy Programming (FP) technique in terms of the Trace (See Sukhatme et al. (1984)). The trace while FP technique is used is $4.387880+8.390343=12.778223$ and that for FGP technique is $4.492375+8.512760=12.619975$. Which gives a relative increase in Trace as $\frac{12.778223-12.619975}{12.619975} \times 100 \% \cong 1.25 \%$ when FP technique is used.

At Phase-II the relative increase in the trace is $\frac{6.50342-6.123896}{6.123896} \times 100 \% \cong 6.20 \%$ when FGP technique is use. Thus we conclude that the use of FP technique is more advisable because it gives a comparative large gain at Phase-II at the cost of a small loss in precision at Phase-I.

[1]. References

[2]. Sukhatme, P.V., Sukhatme, B.V., Sukhatme, S., Asok, C.: Sampling Theory of Surveys with Applications. Iowa State University Press, Iowa, U.S.A. and Indian Society of Agricultural Statistics, New Delhi, India, 1984.

[3]. Geary, R.C.: Sampling methods applied to Irish agricultural statistics. Technical Series, Central Statistical office, Dublin, 1949.

[4]. Dalenius, T.: Sampling in Sweden: Contributions to the Methods and Theories of Sample Survey Practice. Almqvist and Wiksell, Stockholm, 1957.

[5]. Ghosh, S.P.: A note on stratified random sampling with multiple characters. Calcutta Statist. Assoc. Bull. 8, 81-89, 1958.

[6]. Yates, F.: Sampling methods for Censuses and Surveys. Charles Griffin \& Co. Ltd., London, 1960.

[7]. Aoyama, H.: Stratified random sampling with optimum allocation for multivariate populations. Ann. Inst. Statist. Math. 14, 251$258,1963$.

[8]. Folks, J.L., Antle, C.E.: Optimum allocation of sampling units to the strata when there are $R$ responses of interest. J. Amer. Statist. Assoc. 60, 225-233, 1965.

[9]. Chatterjee, S.: A note on optimum allocation. Scand. Actuar. J. 50, 40-44, 1967.

[10]. Chatterjee, S.: Multivariate stratified surveys. J. Amer. Statist. Assoc. 63, 530-534, 1968. 
[11]. Kokan, A.R., Khan, S.U.: Optimum allocation in multivariate surveys: An analytical solution. J. Roy. Statist. Soc. B 29, 115-125, 1967.

[12]. Ahsan, M.J.: A procedure for the problem of optimum allocation in multivariate stratified random sampling. Aligarh Bull. Math. 56, 37-42, 1975-1976.

[13]. Ahsan, M.J.: Allocation problem in multivariate stratified random sampling. J. Indian Statist. Assoc. 16, 1-5 , 1978.

[14]. Ahsan, M.J., Khan, S.U.: Optimum allocation in multivariate stratified random sampling using prior information. J. Indian Statist. Assoc. 15, 57-67, 1977.

[15]. Jahan, N., Khan,M.G.M., Ahsan, M.J.: A generalized compromise allocation. J. Indian Statist. Assoc. 32, 95-10, 1994.

[16]. Jahan, N., Khan, M.G.M., Ahsan, M.J.: Optimum compromise allocation using dynamic programming. Dhaka Univ. J. Sci. 49(2), 197-202, 2001.

[17]. Khan, M.G.M., Khan, E.A., Ahsan, M.J.: An optimal multivariate stratified sampling design using dynamic programming. Aust. N. Z. J. Stat. 45(1), 107-113, 2003.

[18]. Khan, M.G.M., Khan, E.A., Ahsan, M.J.: Optimum allocation in multivariate stratified sampling in presence of non-response. J. Indian Soc. Agricultural Statist. 62(1), 42-48, 2008.

[19]. Singh, S.: Advanced Sampling Theorywith Applications: howMichael 'selected'Amy.Kluwer, Dordrecht , 2003.

[20]. Díaz-García, J.A., Cortez, L.U.: Optimum allocation in multivariate stratified sampling: multi-objective programming. Comunicación Técnica No. I-06-07/28-03-2006 (PE/CIMAT), México, 2006.

[21]. Díaz-García, J.A., Cortez, L.U.: Multi-objective optimisation for optimum allocation in multivariate stratified sampling. Surv. Methodol. 34(2), 215-222, 2008.

[22]. Kozak, M.: Multivariate sample allocation: application of random search method. Statistics in Transition. 7(4), 889-900, 2006.

[23]. Kozak, M.: On sample allocation in multivariate surveys. Comm. Statist. Simulation Comput. 35(4), 901-910, 2006.

[24]. Hansen,M.H., Hurwitz,W.N.: The problem of nonresponse in sample surveys. J. Amer. Statist. Assoc. 41, $517-529,1946$.

[25]. Okafor, F.C.: On double sampling for stratification with sub-sampling the non respondents. Aligarh J. Statist. 14, 13-23, 1994.

[26]. Najmussehar, Bari, A.: Double sampling for stratification with sub-sampling the non-respondents: a dynamic programming approach. Aligarh J. Statist. 22, 27-41, 2002.

[27]. Varshney, R., Najmussehar, Ahsan, M. J.: An optimum multivariate stratified double sampling design in non-reponse. Springer, Optimization letters. Verlag, 2011

[28]. Haseen, S., Iftekhar, S., Ahsan, M. J. and Bari, A.:A fuzzy approach for solving double sampling design in presence of nonresponse. International Journal of Engineering Science and Technology. 4. 2542 - 2551, 2012.

[29]. Charnes, A., Cooper, W. W. : Management Models and Industrial Applications of Linear Programming. Wiley, New York, 1961.

[30]. Bellman, R. E., Zadeh, L. A.: Decision-Making in a Fuzzy Environment. Management Sciences. 17. B141 - B164, 1970.

[31]. Zimmermann, H. J.: Fuzzy Programming and Linear Programming with Several Objective Functions. Fuzzy Sets and System. 1. 45-55, 1978.

[32]. Narasimhan, R.: On Fuzzy Goal Programming - Some Comments. Decision Sciences.11. 532 - 538, 1980. Pal, B. B., Moitra, B. N., Maulik, U.: A Goal Programming Procedure for Fuzzy Multiobjective Linear Fractional Programming Problem. Fuzzy Sets and Systems. 139. $395-405,2003$.

[33]. Biswas, A., Pal, B. B.: Application of Fuzzy Goal Programming Technique to Land Use Planning in Agricultural System.Omega. 33. $391-398,2005$

[34]. Parra, M. A., Terol, A. B., Uria, M. V. R.: A Fuzzy Goal Programming Approach to Portfolio Selection. European Journal of operational Research. 133. $287-297,2001$.

[35]. Miller, C. E.: The Simplex Method for Local Separable Programming. Recent Advances in Mathematical programming (R.L. Groves and P. Wolfe Eds.). 89-100, 1963.

[36]. Cox, M. G.: An Algorithm for Approximating Convex Function by Means of the First Degree Splines. Computer Journal. 14. 272$275,1971$.

[37]. Lin, C. C., Chen, A. P.: Generalization of Yang et al.’s Method for Fuzzy Programming with Linear Membership Functions. Fu zzy Sets and Systems. 132. 347-352, 2002.

[38]. Chang, C. T.: An Efficient Linearization Approach for Mixed Integer Problems. European Journal of Operational Research. 123. 652-659, 2000

[39]. Bazaraa, M. S., Sherali, H. D., Shetty, C. M.: Nonlinear Programming Theory and Algorithms. John Wiley \& Sons, Neywork, 1993.

[40]. Aoyama, H.: Stratified Random Sampling with Optimum Allocation for Multivariate Populations. Annals of the Institute of Statistical Mathematics. 14. 251-258, 1963.

[41]. Ghufran S., Khowaja S., Ahsan M.J.: Optimum Multivariate Stratified Sampling Design with Travel Cost: A Multiobjective Integer Nonlinear Programming Approach. Communications in Statistics- Simulation and Computation. 41. 598-610, 2012.

[42]. Khowaja, S.: Unpublished Ph.D. Thesis of Ms. Saman Khowaja, Department of Statistics \& Operations Research, Aligarh Muslim University, Aligarh,India, Chapter 4. 92-130. 